Research, part of a Special Feature on Catastrophic Thresholds, Perspectives, Definitions, and Applications

\title{
Variability in Population Abundance is Associated with Thresholds between Scaling Regimes
}

\author{
Donald Wardwell $^{1}$ and $\underline{\text { Craig R. Allen }}^{1,2}$
}

\begin{abstract}
Discontinuous structure in landscapes may result in discontinuous, aggregated species bodymass patterns, reflecting the scales of structure available to animal communities within a landscape. The edges of these body-mass aggregations reflect transitions between available scales of landscape structure. Such transitions, or scale breaks, are theoretically associated with increased biological variability. We hypothesized that variability in population abundance is greater in animal species near the edge of bodymass aggregations than it is in species that are situated in the interior of body-mass aggregations. We tested this hypothesis by examining both temporal and spatial variability in the abundance of species in the bird community of the Florida Everglades sub-ecoregion, USA. Analyses of both temporal and spatial variability in population abundance supported our hypothesis. Our results indicate that variability within complex systems may be non-random, and is heightened where transitions in scales of process and structure occur. This is the first explicit test of the hypothetical relationship between increased population variability and scale breaks.
\end{abstract}

Key Words: body mass; Breeding Bird Survey; Everglades; phase transition; scale; textural discontinuity hypothesis

\section{INTRODUCTION}

Ecosystems are complex adaptive systems possessing emergent properties, such as resilience, and discontinuous structure that varies across scales. Ecological processes are scale specific in their effects and create heterogeneous landscapes with scale-specific structure and pattern (Holling 1992). This structure has been described as a dynamic, nested set of adaptive cycles, termed a panarchy (Fig. 1; Holling and Gunderson 2002). Each cycle operates over a discrete range of scale in both time and space and is connected to adjacent levels (adaptive cycles). Ecosystems are structured by key ecological processes that operate at discrete ranges of scale, and ecological organization abruptly shifts with changes in scale. For example, climate affects entire regions over large temporal scales, whereas fire disturbance may affect only portions of a landscape at much smaller spatial scales. The scales upon which key processes occur in landscapes are reflected in discontinuous patterns of structure and resource distribution. Connectivity between adaptive cycles in a panarchy may be from levels above or below, which differs from the topdown control of traditional hierarchical structure.

Adaptive cycles, and structuring processes, are separated from one another by large gaps, so that variables within systems are distributed discontinuously. Discontinuities in animal body-mass distributions reflect discontinuities in scales of structure and process, and may be analogous to thresholds or transition points exhibiting high variability between two ranges of scale (Allen et al. 1999). Animals with body masses that place them in these discontinuities may not be adaptive to the landscape, as there is no ecological structure or resource pattern with which they can interact (Allen and Saunders 2002). In complex systems, increased variability may be associated with pending regime shifts, such as an oligotrophic lake transforming into a eutrophic lake (Carpenter and Brock 2006), or the edge of the range of a stable equilibrium before a transition to a new 
Fig. 1. A panarchy. Three selected levels of a panarchy are illustrated, to emphasize the two connections that are critical in creating and sustaining adaptive capability. One is the "revolt" connection, which can cause a critical change in one cycle to cascade up to a vulnerable stage in a larger and slower one. The other is the "remember" connection, which facilitates renewal by drawing on the potential that has been accumulated and stored in a larger, slower cycle. The number of levels in a panarchy varies, is usually rather small, and corresponds to levels of scale present in a system. (Excerpted from L. H. Gunderson and C. S. Holling, editors. Panarchy: understanding transformations in human and natural systems. Copyright (C) 2001 by Island Press.)

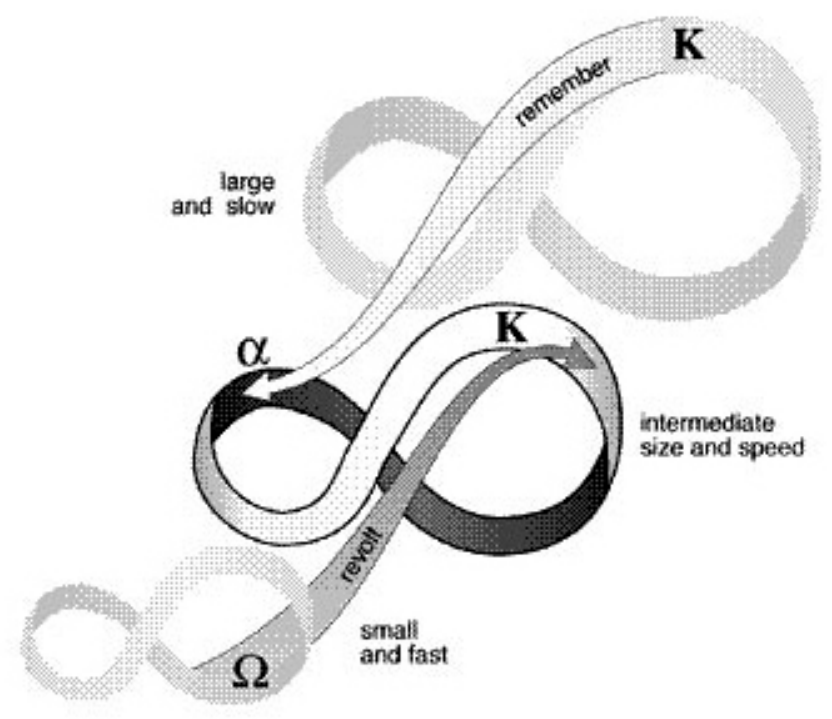

domain (O'Neill et al. 1989). This has been documented for systems undergoing changes of regime (Kleinen et al. 2003, van Nes and Scheffer 2003, Oborny et al. 2005). There may be heightened variability of parameters proximate to discontinuities within systems as well, where discontinuities mark thresholds from one set and scale of controlling processes to another. Allen et al. (1999) hypothesized that biological phenomena associated with greater variability would be more likely to occur at discontinuities. In ecological systems, the analysis of body-mass distributions has been used to determine ecological discontinuities because body mass is an integrative variable allometric with many ecological attributes (Peterson et al. 1998). Allen et al. (1999) found that extinctions and successful invasions were, more often than expected, associated with the edge of body-mass aggregations in the Florida Everglades and other systems. Allen and Saunders $(2002,2006)$ found that nomadic birds were more likely to occur at the edges of body-mass aggregations, and that distance to discontinuity in body-mass pattern was a better predictor of nomadism than diet. Nomadism is often linked with ecosystems that exhibit high variability in resource abundance in time and space.

Populations that exhibit higher variability may be more prone to extinction than those with lower variability (Pimm 1991, Belovsky et al. 1999, Vucetich et al. 2000). Given this and the evidence that populations situated at the edges of body-mass aggregations are also prone to extinction, Allen et al.'s (1999) model may be expanded to suggest that animals with body masses that place them close to discontinuities will exhibit higher variability in abundance than animals with body masses in the interior of body-mass aggregations. Increased environmental variability may enhance the effects of competition, amplifying the extinction 
probability (May 1973, Abrams 1999). May (1973) suggests that even a small amount of environmental variability may have a dramatic effect.

Understanding the role body-mass pattern plays in population variability could have important implications for conservation. Greater environmental variability at discontinuities may be reflected in increased variability in the abundance of species in both space and time. If it were possible to predict which species are likely to be more variable in abundance, then we might be able to determine which species are at an increased risk of extinction. Here, we examine temporal and spatial variability in avian population abundance in relation to a species' position relative to discontinuities in the body-mass distribution of Everglades birds. We hypothesize that species that are at or near the edge of body-mass aggregations will exhibit greater temporal and spatial variability in population abundance than birds with body masses that place them in the interior of body-mass aggregations.

\section{METHODS}

Species distribution and body-mass estimates were determined for the avian fauna of the Florida Everglades sub-ecoregion using published data (Allen et al. 1999). Only species that had established breeding populations in the Everglades were included. Non-indigenous species were not included. Pelagic birds were also excluded because they interact with their environment differently than terrestrial species. In all cases, adult male and female body masses were averaged. We also used the discontinuity analyses published in Allen et al. (1999). That analysis was based on Gap Rarity Index (Restrepo et al. 1997) and was confirmed with split moving-window boundary (Webster 1978, Ludwig and Cornelius 1987) and hierarchical cluster analyses (SAS Institute 1999). The Gap Rarity Index uses simulations to compare observed bodymass distributions with a null distribution established by estimating a continuous unimodal kernel distribution of the log-transformed data (Silverman 1981). Gaps are defined as areas between successive body masses that significantly exceed the discontinuities generated by the null distribution. A species aggregation is a grouping of three or more species with body masses not exceeding the expectation of the null distribution. Species with body masses defining the upper and lower edges of an aggregation have a distance to edge of zero. Distance to edge is determined for other species relative to the mass of the nearest edge defining species.

Population abundances were determined for Everglades birds using Breeding Bird Survey (BBS) data. We selected seven BBS routes (Devil's Garden, Flamingo, Holey Land, Homestead, Pinecrest, Sunniland, and Weaver Station; BBS 2005) from the Everglades sub-ecoregion (Broward, Collier, Hendry, Lee, Miami-Dade, Monroe, and Palm Beach counties) in Florida that had data from 1999 to 2003 (Fig. 2). This time-span was the most recent 5-year period of data available at the inception of our study.

We used the same species list for each route; however, the same birds were not always present on each route. For each species, the coefficient of variation $(\mathrm{CV})$ was determined for each route over the 5-year study period. We used CV because it allowed us to compare the variation of bird populations, which often exhibit substantially different means. We also ascertained CVs of each species across all seven routes for each year to evaluate differences in spatial variability of population abundance. The CVs of edge species were compared with those of interior species using distance-to-edge as the metric of analysis. Distanceto-edge is a measure of a species' proximity (in $\log _{10}$ body mass) to the edge of its body-mass aggregation. Those species directly on the edge of an aggregation have a distance-to-edge of zero. For interior species, the distance was measured to the closest edge (Fig. 3). There were cases where a species was observed in only 1 year of our temporal analyses. In these cases, CVs were calculated to be 223.6, regardless of the number of individuals detected in the 1 year. Similarly, there were cases in the spatial analyses where species were detected at only one site, yielding a CV of 264.6 regardless of the number of individuals detected at the one site. We did not consider these cases in our analyses of spatial and temporal abundance.

We used a generalized linear model with normal error distribution for analysis of temporal variability in population abundance. In our model, $\mathrm{CV}$ was the dependent variable and distance-to-edge was the independent variable. A generalized linear model with normal error distribution also was employed to evaluate spatial variability in population 
Fig. 2. Map of southern Florida with Breeding Bird Survey routes used for this study. Routes include Devil's Garden, Holey Land, Homestead, Pinecrest, Sunniland, Weaver's Station, and Flamingo.

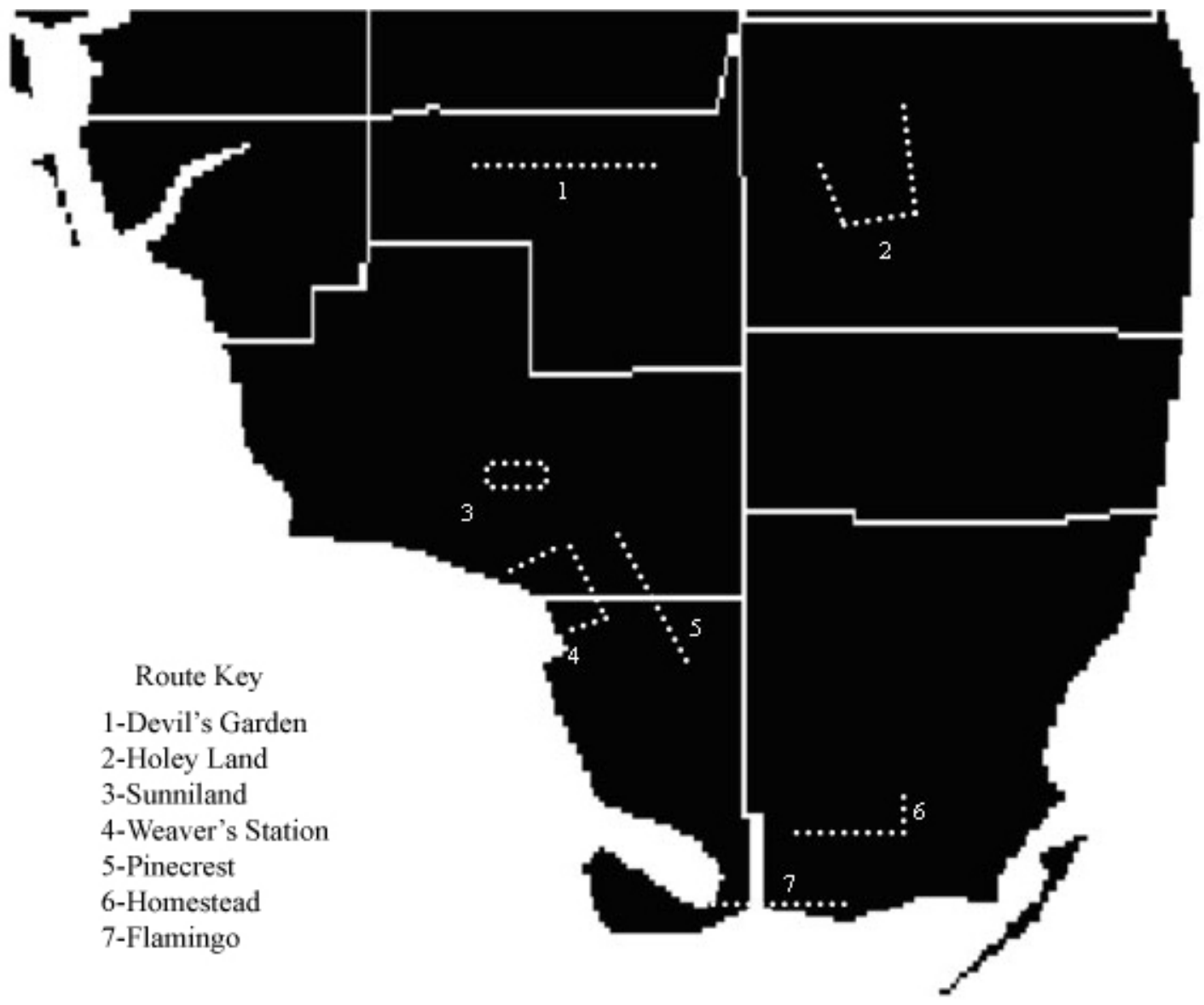

abundance. Coefficient of variation was the dependent variable and distance-to-edge was the independent variable. We used SAS (Statistical Analysis System) version 9.1 (@2002-2003) for our generalized linear model analyses.

\section{RESULTS}

The Everglades bird species distribution was discontinuous, and consisted of 13 body-mass aggregations defined by 12 discontinuities (Allen et al. 1999; Appendix 1). The BBS provided abundance data for 97 of 106 birds in the Florida Everglades species list. Many of the 97 species for which abundance data were collected were not present at all seven routes. Coefficients of variation for these species were determined for the routes with abundance data, which in some cases was for only one route (Appendix I).

There were outliers in the data (Fig. 4). The bluegray gnatcatcher (Polioptila caerulea), prairie warbler (Dendroica discolor), and northern parula (Parula americana) exhibited relatively extreme distances to the edge of their body-mass aggregation (distances were greater than 0.14). Therefore, these outliers were removed from the data associated with these species and the analyses were performed with the truncated data. 
Fig. 3. Possible relationships between coefficient of variation (CV) in population abundance in time and space and position of species body mass relative to discontinuities. A) CV is larger in species proximal to discontinuities. B) CV is smaller in species proximal to discontinuities. C) CV is random with respect to proximity to discontinuities.

A.

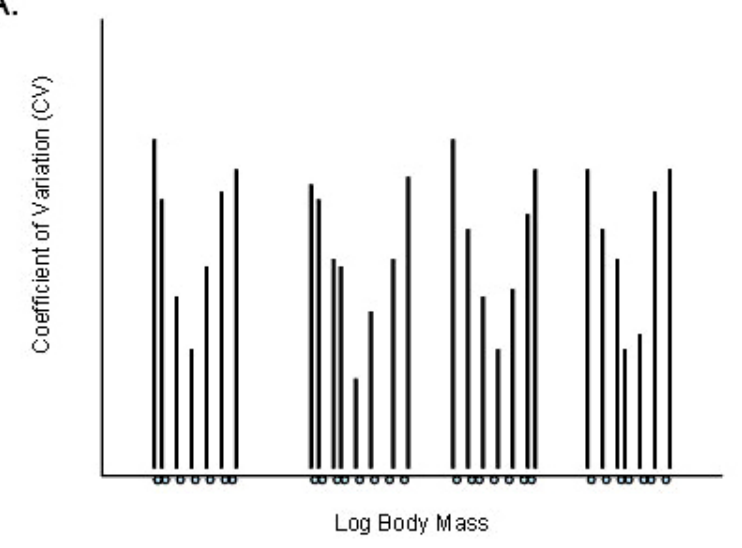

B.

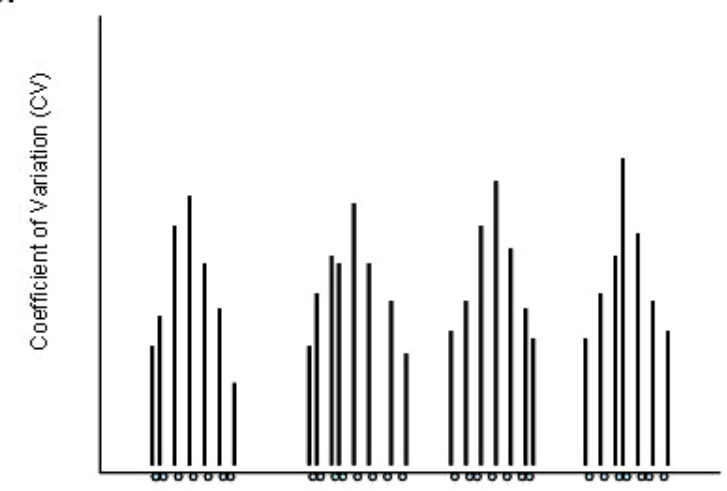

Log Body Mass

C.

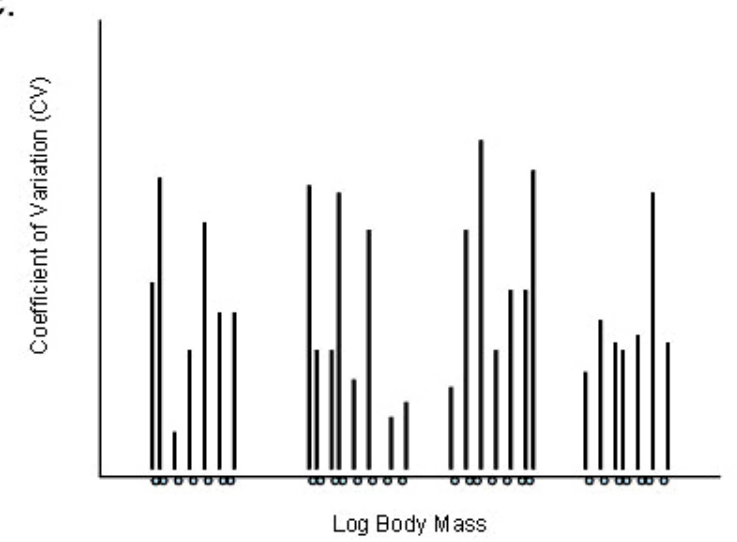


Fig. 4. Plots of coefficient of variation (CV) in relation to distance-to-edge (edge) for temporal analysis (A) and spatial analysis (B) of bird species of the Florida Everglades sub-ecoregion. Each point (diamond) represents one species at one BBS route and their respective CV and edge. Outliers (dashed square) and data removed due to insufficient observations (oval) are highlighted.
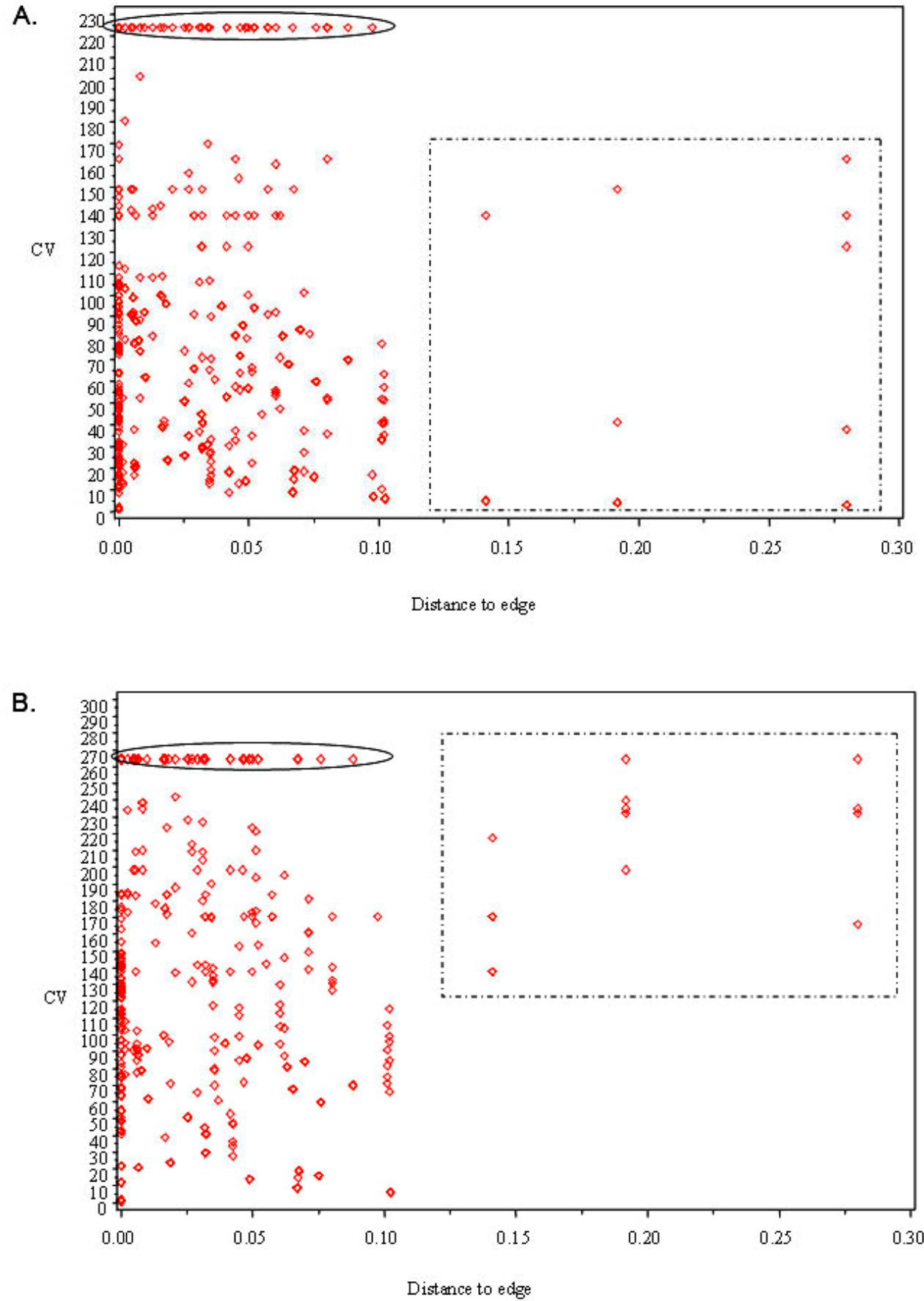
Table 1. ANOVA table from temporal analysis of the relationship between coefficient of variation and distance-to-edge of body-mass aggregations (A) and spatial analysis of the relationship between coefficient of variation and distance-to-edge of body-mass aggregations (B) of bird species of the Florida Everglades sub-ecoregion. Includes source, degrees of freedom (DF), Sum of squares (SS), Mean squares (MS), F values, associated probability values, as well as estimates of "distance-to-edge" and associated standard errors.

\begin{tabular}{lccccc}
\hline \hline Source & DF & SS & MS & F Value & Pr $>$ F \\
\hline A. & 1 & 17269.6 & 17269.6 & 11.64 & $<0.001$ \\
Model & 441 & 654312.0 & 1483.7 & & \\
Error & 442 & 671581.6 & & & \\
Total & & Standard error $=60.7$ & & \\
Estimate of "edge" = -207.1 & & & & \\
B. & & & & \\
Model & 1 & 28122.2 & 28122.2 & 9.35 & \\
Error & 333 & 1001776.7 & 3008.3 & & \\
Total & 334 & 1029898.9 & & \\
Estimate of "edge" = -303.9 & Standard error $=99.4$ & & \\
\hline
\end{tabular}

An effect $(\mathrm{F}=11.64 ; \mathrm{df}=1,441 ; P=<0.001)$ of distance-to-edge was demonstrated in the analysis of temporal variability (Table 1A). The species exhibiting larger $\mathrm{CVs}$ are closer to their respective aggregation edges (Fig. 5). Conversely, species that are furthest from the edge of their aggregations exhibit relatively low CVs. Analysis of spatial variability in population abundance also revealed a statistically significant effect $(\mathrm{F}=9.35 ; \mathrm{df}=1,333$; $P=0.002$; Table 1B) of distance-to-edge. The linear regression of the data reveals a similar pattern to that of the temporal variability analysis - a negative relationship between $\mathrm{CV}$ and distance-to-edge (Fig. 6). In fact, the slope is steeper than in the temporal analysis. These results both support the model presented in Fig. 3(A). Variation in abundance, in both time and space, is non-randomly distributed in terms of discontinuities in body-mass distributions. Species with masses that place them closer to discontinuities have more variable populations.

\section{DISCUSSION}

Analysis of both temporal and spatial variability in population abundance supports our hypothesis that variation is increased at the gaps in body-mass distributions that represent abrupt changes in scale. There is a relationship between variability and distance to the edge of body-mass aggregations (Figs. 5 and 6), evidence that, in the Everglades' bird community, species with body masses nearer to discontinuities are more likely to have fluctuating abundance in time and space than other species. This study examined trends across the whole range of scales; however, it may be of interest to determine scale-specific relationships between variability and location within a scale or body-mass aggregation.

Allen et al. (1999) proposed that biological phenomena associated with greater environmental variability (e.g., nomadism and invasion/exinction) 
Fig. 5. Plot of coefficient of variation (CV) in relation to distance-to-edge (edge) of truncated data for temporal analysis of bird species of the Florida Everglades sub-ecoregion. Each point (diamond) represents one species at one BBS route and their respective CV and edge. The regression line (solid line) is accompanied by its $95 \%$ confidence limits (dashed line).

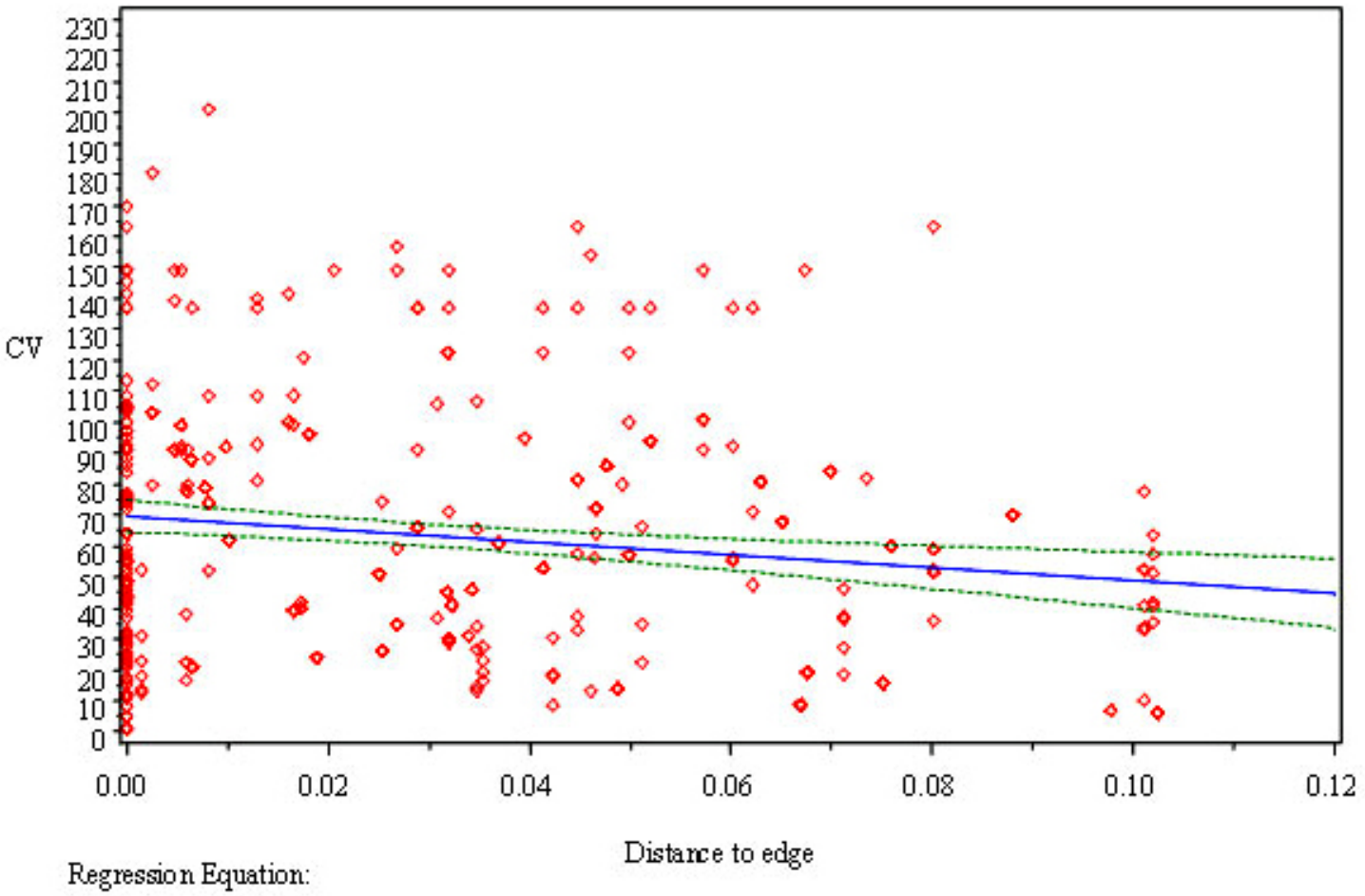

$\mathrm{CV}=69.6648-207.1383^{*}$ distance to edge

are more likely to occur in species located near the edges of body-mass aggregations. Subsequent research has supported their contention (Allen and Saunders 2002, 2006-nomadism; Skillen and Maurer 2007-decline; C. R. Allen, unpublished data-migration), but the idea that the edges of body-mass aggregations are associated with greater variability has not been explicitly tested. These gaps and edge areas may represent scale breaks, where it has been hypothesized that high variability should exist in biological systems (Wiens 1989). Our analyses measure environmental variability using population abundance. The results provide evidence that spatial and temporal variability is greater with increased proximity to discontinuities.

We detected a relationship between $\mathrm{CV}$ of population abundance and distance to discontinuities despite some inherent weaknesses in our analyses. Nine of the species had no data at all and many others had data for some of the BBS routes, but not all seven. It may be that certain species have a limited range within the Everglades and, thus, would not occur in all of the routes we employed. There may be inherent biases in the collection methods of BBS data (Sauer et al. 1994) and the uncertainty of the species distribution (Allen et al. 1999). Minimal detectability of interior habitat species, observer differences, and limited surveys (one per route per year) are problems associated with BBS data (Sauer et al. 1994). Imperfect detectability of these surveys has several potential effects on CV. Population means approaching zero are more sensitive to changes in standard deviation. For example, some of the species in our study were detected in only 1 year of the 5-year period of our temporal analyses. 
Fig. 6. Plot of coefficient of variation (CV) in relation to distance-to-edge (edge) of truncated data for spatial analysis of bird species of the Florida Everglades sub-ecoregion. Each point (diamond) represents one species during 1 year and their respective CV and edge. The regression line (solid line) is accompanied by its $95 \%$ confidence limits (dashed line).

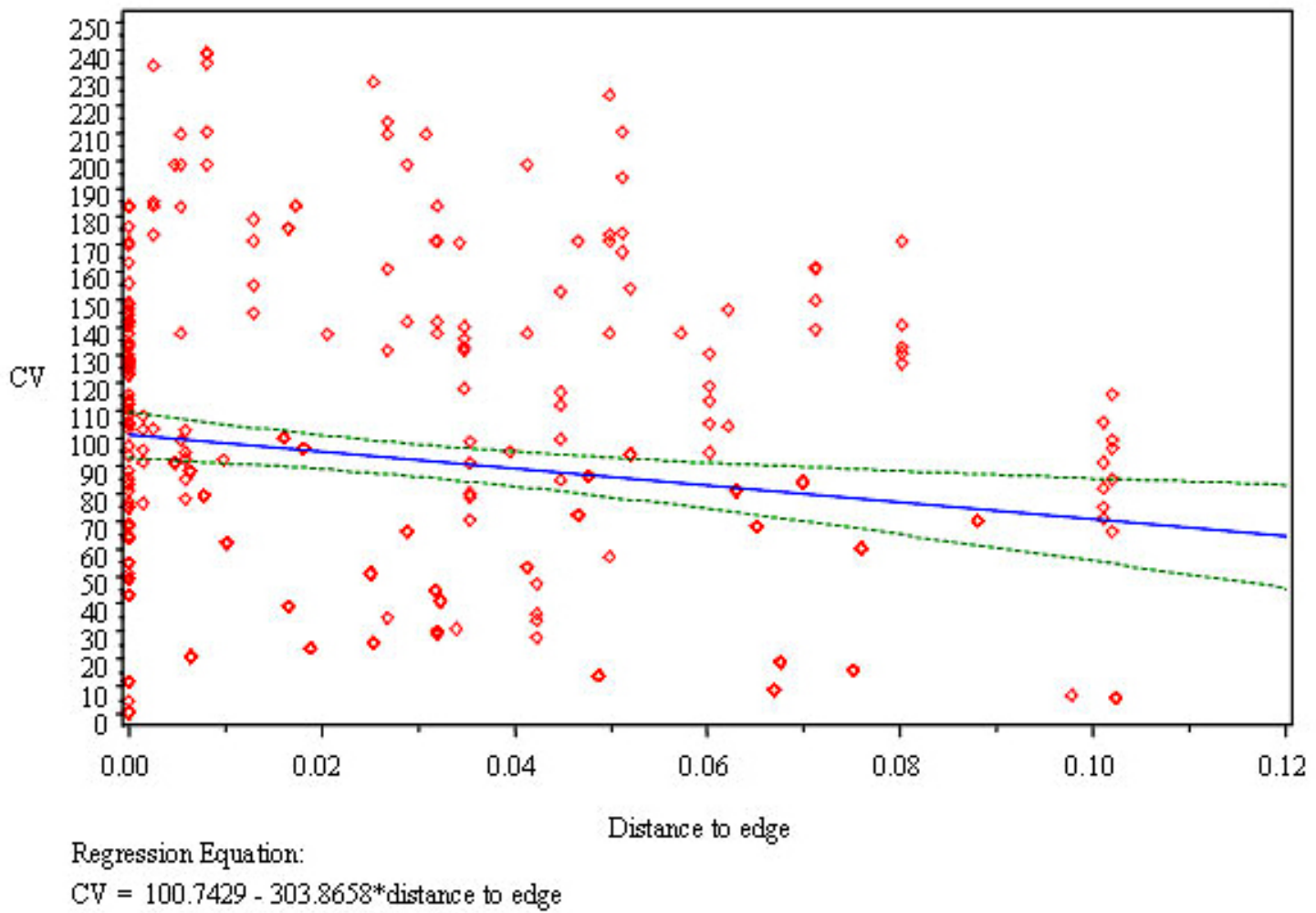

In these cases, CVs were calculated to be 223.6, regardless of the number of individuals detected in the 1 year. Also, observer differences may be increasing CVs. Less experienced observers may be counting less efficiently than more experienced observers, creating great variation between counts. These and other imperfections of BBS detectability may be clouding our ability to detect an even stronger relationship between variability in population abundance and CV. The distribution we used (published in Allen et al. 1999) excludes nonindigenous species. Inclusion of non-indigenous species, which are more likely to be located at bodymass aggregation edges (Allen et al. 1999) and more likely to have variable populations, may produce different results. Finally, we use only one community in these analyses and we measure variability over a relatively short 5-year time period. Testing our hypothesis in more communities and across longer periods of time would likely improve our understanding of the relationship between scale changes and variability in population abundance.

Locating within-system thresholds also may provide an alternative method of identifying discontinuities in data such as body-mass distributions. Currently, there are several methods employed in determining discontinuities in animal body-mass distributions, including Gap Rarity Index (Restrepo et al. 1997), Cluster analysis (SAS Institute 1999), Bayesian CART analyses (Chipman et al. 1998, Bremner and Taplin 2004), and kernel density estimation (Havlicek and Carpenter 2001). Using data independent of body mass, such as 
population variability, to identify discontinuities and cross-scale structure may increase the robustness of discontinuity analyses and assuage some of the earlier criticisms leveled at the detection of structure in body-mass distributions (Manly 1996, Siemann and Brown 1999).

Carpenter and Brock (2006) demonstrated that increasing variance is an indicator of impending ecological transitions. They suggest that certain parameters of complex systems become more variable as they approach thresholds that occur when lakes change state. We show that variability within complex systems may be non-random, and is heightened where shifts in scales of process and structure occur, that is, where within-system thresholds occur. This suggests that it is possible to identify those variables that are most likely to exhibit increased variability before regime shifts. This would greatly simplify monitoring established to determine when there is an increased probability of changing the state of a system.

Responses to this article can be read online at: http://www.ecologyandsociety.org/voll4/iss2/art42/ responses/

\section{Acknowledgments:}

The Nebraska Cooperative Fish and Wildlife Research Unit is jointly supported by a cooperative agreement between the United States Geological Survey, the Nebraska Game and Parks Commission, the University of Nebraska, the United States Fish and Wildlife Service, and the Wildlife Management Institute.

\section{LITERATURE CITED}

Abrams, P. A. 1999. Is predator-mediated coexistence possible in unstable systems? Ecology 80:608-621.

Allen, C. R., E. A. Forys, and C. S. Holling. 1999. Body mass patterns predict invasions and extinctions in transforming landscapes. Ecosystems 2:114-121.
Allen, C. R., and D.A.Saunders. 2002. Variability between scales: predictors of nomadism in birds of an Australian Mediterranean-climate ecosystem. Ecosystems 5:348-359.

Allen, C. R., and D. A. Saunders. 2006. Multimodel inference and the understanding of complexity, discontinuity, and nomadism. Ecosystems 9:694-699.

Belovsky, G. E., C. Mellison, C. Larson, and P. A. van Zandt. 1999. Experimental studies of extinction dynamics. Science 286:1175-1177.

Breeding Bird Survey. 2005. Raw BBS data search тепи. United States Geological Survey, Washington, D.C., USA. [online] URL: http://www.pwrc.usgs.gov/ bbs/retrieval/menu.cfm.

Bremner, A. P., and R. H. Taplin. 2004. Performance of a localized tree splitting criterion in tree averaging. Australia and New Zealand Journal of Statistics 46:583-599.

Carpenter, S. R., and W. A. Brock. 2006. Rising variance: a leading indicator of ecological transition. Ecology Letters 9:311-318.

Chipman, H. A., E. L. George, and R. E. McCulloch. 1998. Bayesian CART model search: rejoinder. Journal of American Statistical Association 93:957-960.

Havlicek, T. D., and S. R. Carpenter. 2001. Pelagic species size distributions in lakes: are they discontinuous? Limnology and Oceanography 46:1021-1033.

Holling, C. S. 1992. Cross-scale morphology, geometry, and dynamics of ecosystems. Ecological Monographs 62:447-502.

Holling, C. S., and L. H. Gunderson. 2002. Panarchy: understanding transformations in human and natural systems. Island Press, Washington, D.C., USA.

Kleinen, T., H. Held, and G. Petschel-Held. 2003. The potential role of spectral properties in detecting thresholds in the earth system: application to the thermohaline circulation. Ocean Dynamics 53:5363. 
Ludwig, J.A., and J. M. Cornelius. 1987. Locating discontinuities along ecological gradients. Ecology 68:448-450.

Manly, B. F. J. 1996. Are there clumps in bodysize distributions? Ecology 77:81-86.

May, R. M. 1973. Stability in randomly fluctuating versus deterministic environments. The American Naturalist 107:621-650.

Oborny, B., G. Meszena, and G. Szabo. 2005. Dynamics of populations on the verge of extinction. Oikos 109:291-296.

O'Neill, R. V., A. R. Johnson, and A. W. King. 1989. A hierarchical framework for the analysis of scale. Landscape Ecology 3:193-205

Peterson G., C. R. Allen, C. S. Holling. 1998. Ecological resilience, biodiversity, and scale. Ecosystems 1:6-18.

Pimm, S. L. 1991. Balance of nature? The University of Chicago Press, Chicago, Illinois, USA.

Restrepo C., L. M. Renjifo, and P. Marples. 1997. Frugivorous birds in fragmented neotropical montane forests: landscape pattern and body mass distribution. Pages 171-189 in W. F. Laurance, R. O. Bierregaard, and C. Moritz, editors. Tropical forest remnants: ecology, management and conservation of fragmented communities. University of Chicago Press, Chicago, Illinois, USA.

SAS Inststitute Inc. 1999. SAS/STAT user's guide. Version 8. Volume 2. SAS Institute Inc., Cary, North Carolina, USA.

Sauer, J. R., B. G. Peterjohn, and W. A. Link. 1994. Observer differences in the North American Breeding Bird Survey. The Auk 111:50-62.

Siemann, E., and J. H. Brown. 1999. Gaps in mammalian body size distributions reexamined. Ecology 80:2788-2792.

Silverman, B. W. 1981. Using kernel density estimates to investigate multimodality. Journal of the Royal Statistical Society B 43:97-99.

Skillen, J. J., and B. A. Mauer. 2007. The ecological significance of discontinuities in body mass distributions. In C. R. Allen and C. S. Holling, editors. Discontinuity theory. University of Chicago Press, Chicago, Illinois, USA. In press.

van Nes, E. H., and M. Scheffer. 2003. Alternative attractors may boost uncertainty and sensitivity in ecological models. Ecological Modelling 159:117124.

Vucetich, J. A., T. A. Waite, L. Qvarnemark, and S. Ibarguen. 2000. Population variability and extinction risk. Conservation Biology 14:17041714.

Webster, R. 1978. Optimally partitioning soil transects. Journal of Soil Science 29:388-402.

Wiens, J. A. 1989. Spatial scaling in ecology. Functional Ecology 3:385-97. 
Appendix 1. Species body mass (body mass, in $\log 10$ grams), distance to edge (Distance), aggregation membership (Aggregation), temporal variance 1999-2003 at each BBS routes (Sunlnd = Sunniland; DevGdn = Devil's Garden; HlyLnd = Holey Land; WvrStn = Weaver Station; Flmngo = Flamingo; Hmstd $=$ Homestead; Pncrst $=$ Pinecrest $)$, and spatial variance across all BBS routes within years. 